\title{
Liderazgo pedagógico: autopercepciones de directivos en la gestión de las instituciones educativas estatales
}

\author{
Pedagogical leadership: self-perceptions of managers in the \\ management of public educational institutions
}

\section{Gabriel Adalberto Vela-Quico' ${ }^{1}$ y Telmi Janet Cáceres-Coaquira²}

Revista

Educación y Sociedad

Citar como: Vela-Quico, G. A. y Cáceres-Coaquira, T. J. (2020). Liderazgo pedagógico: autopercepciones de directivos en la gestión de las instituciones educativas estatales. Revista Educación y Sociedad, 01(02), 44-54. doi: $10.53940 /$ reys.v1i2.58

Artículo recibido: 12-05-2020 Artículo aprobado: 10-10-2020 Arbitrado por pares

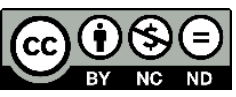

\section{ACEES}

\section{Resumen}

Una de las prioridades para el mejoramiento de una institución educativa es el liderazgo pedagógico de los gestores escolares. En ese sentido, el propósito de la investigación fue analizar cómo se auto perciben los directivos respecto a sus competencias prescritas por el Ministerio de Educación. Para este fin se aplicó una escala de autovaloración a 60 directivos de escuelas estatales de Arequipa (Perú). En virtud a esto, los hallazgos revelan limitaciones en las áreas o dimensiones relacionadas con el construir el diagnóstico y en el transitar por la planificación participativa en la gestión. También, los sujetos reconocieron tener problemas en la gestión de la información que produce la institución. Se concluye, por tanto, que las competencias y desempeños directivos no están en el nivel óptimo desde sus propias percepciones evaluativas.

Palabras clave: autovaloración de directores, competencias directivas, liderazgo pedagógico

\section{Abstract}

One of the priorities for the improvement of an educational institution is the pedagogical leadership of school administrators. In this sense, the purpose of the research was to analyze how managers perceive themselves with respect to their competencies prescribed by the Ministry of Education. For this purpose, a self-assessment scale was applied to 60 directors of state schools in Arequipa (Peru). By virtue of this, the findings reveal limitations in the areas or dimensions related to constructing the diagnosis and moving through participatory planning in management. Also, the subjects acknowledged having problems in the information management successfully the information produced by the institution. It is concluded, therefore, that managerial skills and performances are not at the optimal level from their own evaluative perceptions.

Key words: directors self-assessment, management skills, pedagogical leadership 


\section{Introducción}

La sociedad actual tiene muchos retos, uno de ellos es resignificar el papel de la educación en el desarrollo sostenible. La educación debe preocuparse, por tanto, en gestar personas con una conciencia global del mundo, para ello debe asimilar y socializar la cultura, en especial a las nuevas generaciones. El reto de transformar las prácticas formativas del mundo descansa, en gran medida, en los sistemas educativos. No obstante, que esto se cristalice, la educación necesita superar los marcos de la enseñanzaaprendizaje y ver sus desafíos en las reformas educativas que buscan lograr la calidad y equidad. Del mismo modo, y en el terreno de la institucionalidad educativa, se esperaría la articulación de varios factores como el currículo, la infraestructura, las tecnologías de información, las políticas educativas y, sobre todo, el papel transformador de los docentes en calidad de directivos.

Al respecto, la evidencia empírica señala que los directivos con liderazgo pedagógico contribuyen a dinamizar, apoyar y animar el desarrollo dentro de las escuelas; ello implica una decidida participación de todos y cada uno de los miembros de la escuela. Asimismo, cada quien moviliza su iniciativa personal, sus capacidades profesionales y su experiencia laboral en función de objetivos comunes (visión y misión compartida); por ello, se le llama también liderazgo distribuido, compartido, colaborativo, democrático o participativo (Soto, Torres y Barboza, 2019). En este sentido, los líderes -sean formales o no- estarán apostando por la innovación, la autorregulación y la proactividad como elementos claves para la conducción de las instituciones (Ganga-Contreras, Villegas-Villegas, y Pedraja-Rejas, 2016).

También, es importante destacar que la gestión escolar tradicional comprende despliegues que desbordan la autonomía, se abocan a la rendición de cuentas y se centran con frecuencia al control; y en donde se busca trascender de lo administrativo y a un liderazgo para el aprendizaje que vincula su ejercicio con los logros académicos del alumnado y con los resultados del establecimiento educacional. En esta coyuntura, el Informe McKinsey señala que un buen liderazgo escolar es un factor clave en la calidad de la educación, pero este, debe ser de tipo transformador (Barber y Mourshed, 2007; Vela-Quico y Villalba, 2019).

El liderazgo pedagógico se concreta en prácticas concretas denominadas desempeños que, en el caso del Perú, se encuentran normadas en el Marco de Buen Desempeño Directivo - MBDDIR (MINEDU, Marco de Buen Desempeño Directivo, 2014). En este documento, la competencia 1 señala: conduce la planificación institucional a partir del conocimiento de los procesos pedagógicos, el clima escolar, las características de los estudiantes y su entorno, orientándola hacia el logro de metas de aprendizaje. Luego, en el desempeño 2 establece: diseña de manera participativa los instrumentos de gestión escolar teniendo en cuenta las características del entorno institucional, familiar y social, estableciendo así metas de aprendizaje. Esto significa que debe lograr convocar a los actores educativos para formular y reajustar de manera conjunta los instrumentos de gestión escolar; analizando las características de los procesos pedagógicos, el clima escolar, entorno institucional, familiar y social. Además, gestiona el consenso para determinar la visión, la misión y las metas institucionales y de aprendizaje. Esto comprende, el comunicar la naturaleza, el contenido, las tareas y los resultados que contemplan los instrumentos de gestión elaborados para promover la identificación de la comunidad educativa con la escuela.

En ese sentido, el liderazgo pedagógico en la gestión escolar se asume como el conjunto de acciones planificadas y relacionadas entre sí - que movilizan a toda la comunidad educativa organizada-; las mismas que gatillarían sinergias que permitirá alcanzar los resultados esperados en términos del logro de los aprendizajes, el aseguramiento del acceso y la permanencia en la educación básica de los estudiantes. Todas estas expectativas se alcanzarían con el liderazgo pedagógico de los directivos o de quienes haga sus veces (MINEDU, 2019). 
Adicional a lo mencionado, el espíritu de este marco del buen desempeño asume que el liderazgo pedagógico se da en un contexto concreto; una organización o asociación o colectivo. En nuestro caso, ese espacio institucional lo conforman las escuelas en concreto; sin embargo, gran parte de las investigaciones acerca del liderazgo pedagógico provienen del campo de la administración desde una perspectiva que no necesariamente respondería a las particularidades de las escuelas.

De todos modos, la categoría escuela no es un todo uniforme y, por ende, existen diversas tipologías clasificatorias de la misma. Así, Lorenzo (2005) distingue hasta cuatro enfoques de lo que es una escuela Desde el enfoque tecno-racionalista, la escuela es analizada como una organización burocrática, racional y jerárquica, donde la autoridad emana de arriba hacia abajo y donde suelen estar bien definidos los roles y funciones, tanto como los procesos de dirección. Esta forma de ver la escuela no hace distinción con los modelos tradicionales de análisis de organizaciones estrictamente formales; que se caracterizan por un significativo proceso de producción, división del trabajo y poder de la autoridad, descuidando por ello su real dimensión. Por otro lado, desde un enfoque más flexible, se ha catalogado a la escuela como un espacio para el aprendizaje democrático. En ella se da lugar a la práctica de valores como la justicia y la organización en la cual todos los actores tienen un espacio en la toma de decisiones.

También, la escuela puede verse como el lugar de significados valiosos para cada uno de los miembros que interactúan en ella, situándola como un lugar de intercambio cultural, donde emergen las construcciones sociales y los objetivos de cambio. Por último, desde una mirada más crítica, el enfoque político, analiza a la escuela como un conglomerado de intereses entre los sujetos, donde los conflictos entre docentes, padres de familia o tutores, directivos y, en menor medida, los estudiantes surgen en la búsqueda de la satisfacción de intereses personales, grupales e institucionales.

Podemos concluir que cada uno de estos enfoques ayuda a entender a la escuela como una organización multidimensional, singular y compleja. Y, esto, nos lleva a verla desde una óptica político desde el cual se rebate los elementos tradicionales de la teoría de la organización (tales como la autoridad, los roles, los objetivos definidos y la coherencia de la acción) para reflejar desde su especificidad la visión pragmática de su realidad, es decir, la micropolítica de la escuela. Actualmente, se necesita un "líder del aprendizaje" que conforma las condiciones para que todos puedan aprender de forma continua y que trabaja de manera colaborativa para la mejora (Bolívar, Caballero y García-Garnica, 2017).

En ese terreno, hace años se estudió al liderazgo como un rasgo característico de ciertos hombres que tuvieron un lugar importante en la historia (Teoría de los Rasgos de la Personalidad). Posteriormente, la Teoría Conductual distingue entre líderes y seguidores y los diferentes estilos de liderazgo según la actitud de estos últimos. Para la Teoría Situacional, el éxito del líder depende de su carácter personal, formación y de las características concretas de la situación que enfrenta: relaciones líder-miembros, grado de estructuración de la tarea, poder posicional formal del líder. Los principales autores de esta teoría son Hersey y Blanchard con los estilos delegativo, directivo, participativo y tutorial. Finalmente, los estilos de liderazgo que plantean Bass y Avolio y sobre la base de una revisión de la teoría de Burns que propone los estilos transaccional y transformacional. Estos últimos enfoques surgieron desde el campo de la administración de las organizaciones y buscan una alternativa de dirección para favorecer una mayor coherencia de objetivos y productividad de los empleados.

A diferencia de lo mencionado, la teoría sociocrítica del liderazgo sostiene que, a diferencia del estudio tradicional sobre las organizaciones productivas, las escuelas deben ser estudiadas teniendo en cuenta que en ellas coexisten la diversidad de metas, las disputas ideológicas y la diversidad de fuentes de poder, entre otros aspectos que no siempre se visualizan desde otros enfoques de liderazgo. De esta manera, los estilos de liderazgo desde esta posición son más bien expresiones de lucha por la conquista y el mantenimiento de la estabilidad política dentro de la organización; usando para ello recursos como compromisos, negociaciones, transacciones e incluso amenazas, presiones y tratos secretos. 
En consecuencia, las instituciones educativas requieren equipos directivos con alto liderazgo para obtener mejores aprendizajes (Barber y Mourshed, 2007). Los estudios muestran que el liderazgo directivo es la segunda variable más relevante para elevar la calidad de los aprendizajes y sin dejar de valorar la calidad de los docentes. En este contexto, el Ministerio de Educación del Perú está destinando recursos para el desarrollo de capacidades de los directores de escuelas del país para formar, básicamente, directores de tipo técnico y dejando de lado la conquista de competencias blandas. Todo esto se materializa en el Programa Nacional de Formación y Capacitación para Directores y Subdirectores que ofrece un Diplomado y Especialización en gestión escolar con liderazgo pedagógico, que se ubica dentro del seno de la implementación de la Ley de Reforma Magisterial y en consonancia con el "Marco de Buen Desempeño del Directivo" (MINEDU, 2014).

La perspectiva del liderazgo pedagógico implica reconocer que hay varios estilos de liderazgo directivo con claras diferencias. La teoría conductual refiere los estilos autocrático, democrático y permisivo. La perspectiva situacional habla de diversos tipos del liderazgo como el empobrecido, el de tarea, el punto medio, el club de campo y el de equipo. Posteriormente, la teoría transformacional caracteriza el liderazgo transformacional y transaccional (García Garduño, Slater y López Gorosave, 2010). Estas mismas teorías, llevadas al campo educativo, supusieron el surgimiento de nuevas clasificaciones que refieren a los estilos de liderazgo del director. De esta manera, en los años noventa, Leithwood, Begley y Cousins (1990) enunciaron una nueva tipología basada en el elemento educativo focalizado por los directores en su práctica: a) estilo basado en el clima y las relaciones; b) estilo basado en los alumnos; c) estilo basado en los programas; y, d) estilo basado en la administración. En ese camino se ha ido consolidando la concepción de que el liderazgo pedagógico como la labor de movilizar e influenciar a otros para articular y lograr las intenciones y metas compartidas de la escuela. Entonces, el liderazgo es una cualidad de la persona que lo ejerce y también puede constituirse en una característica de la gestión de la institución; en la que personas participan de un proceso, conducido por el director, orientado éxito de los resultados y metas de la organización.

Por otro lado, de las distintas propuestas de modelos de liderazgo (estratégico, sostenible, sirviente, emocional, ético y transaccional, entre otras) las dos más potentes han sido el liderazgo pedagógico, procedente del movimiento de escuelas eficaces y el liderazgo transformativo, vinculado al movimiento de reestructuración escolar (Bolívar, 2010). Bajo este panorama, Bolívar, López y Murillo (2013) señala que hay tres grandes dimensiones actuales de la investigación sobre el liderazgo: el liderazgo pedagógico o para el aprendizaje; el liderazgo distribuido; y, el liderazgo para la justicia social. Vemos así que, en la actualidad, la comprensión del liderazgo se aleja de la visión del director como único agente con poder, para adoptar una perspectiva distribuida o compartida. A su vez, se propugna un liderazgo pedagógico capaz de incidir en la mejora de los aprendizajes, creando condiciones para que el profesorado pueda desplegar de la manera más óptima su misión. Y también, por último, se persigue un liderazgo para la justicia social que asegure una buena educación a todos los alumnos, desde una perspectiva inclusiva y equitativa (Bolívar et al., 2013).

En virtud a lo mencionado, la presente investigación busca indagar en torno a la siguiente interrogante: ¿Cómo se auto valoran los directivos en las competencias que poseen para ejercer el liderazgo pedagógico en instituciones educativas estatales de Arequipa?

\section{Metodología}

La presente investigación es de nivel aplicado y de tipo descriptivo (Hernández, R. Fernández, C. y Baptista, P., 2003). Para este efecto se aplicó - bajo consentimiento informado - un instrumento a 60 directivos, entre directores y sub directores, de instituciones educativas estatales de la región Arequipa (Perú). Estos directivos pertenecían a los diferentes niveles y modalidades del sistema educativo peruano. Además, cabe precisar que estos sujetos ( $48 \%$ hombres y $52 \%$ mujeres) fueron convocados en el marco de 
una capacitación de directivos del Ministerio de Educación. La escala de autovaloración fue elaborada por los autores que suscriben este artículo; la misma que fue validada por juicio de expertos y sometida a una prueba piloto. En cuanto al contenido, este instrumento se diseñó a partir de lo establecido en el MBDDir, tomando dos competencias afines con la planificación y evaluación de la gestión escolar (Tabla 1):

Tabla 1

Matriz de la Escala de Auto Valoración

\begin{tabular}{|c|c|c|}
\hline Competencia & Desempeño & Dimensiones \\
\hline $\begin{array}{l}\text { 1. Conduce la } \\
\text { planificación } \\
\text { institucional a partir del } \\
\text { conocimiento de los } \\
\text { procesos pedagógicos, el } \\
\text { clima escolar, las } \\
\text { características de los } \\
\text { estudiantes y su entorno, } \\
\text { orientándola hacia el } \\
\text { logro de metas de } \\
\text { aprendizajes. }\end{array}$ & $\begin{array}{l}1 \text { Diagnostica las características } \\
\text { del entorno institucional, } \\
\text { familiar y social que influyen en } \\
\text { el logro de las metas de } \\
\text { aprendizaje. } \\
2 \text { Diseña de manera } \\
\text { participativa los instrumentos } \\
\text { de gestión escolar teniendo en } \\
\text { cuenta las características del } \\
\text { entorno institucional, familiar y } \\
\text { social, estableciendo metas de } \\
\text { aprendizaje }\end{array}$ & $\begin{array}{l}\text { - Identifica las características de su institución educativa } \\
\text { - } \text { Realiza un análisis reflexivo de las fortalezas, debilidades y } \\
\text { - Caracteriza las condiciones de su institución para anticipar y } \\
\text { predecir objetivos de mejora } \\
\text { - Convoca a los actores educativos para formular y reajustar de } \\
\text { manera conjunta los instrumentos de gestión escolar } \\
\text { - Establece metas, objetivos e indicadores en función del logro de } \\
\text { aprendizaje de los estudiantes } \\
\text { Comunica y difunde los instrumentos de gestión elaborados para } \\
\text { promover la identificación de la comunidad educativa con la } \\
\text { escuela. }\end{array}$ \\
\hline \multirow[t]{2}{*}{$\begin{array}{l}\text { 4. Lidera procesos de } \\
\text { evaluación de la gestión } \\
\text { de la institución } \\
\text { educativa y de rendición } \\
\text { de cuentas, en el marco } \\
\text { de la mejora continua y el } \\
\text { logro de aprendizajes }\end{array}$} & $\begin{array}{l}\text { 12. Gestiona la información que } \\
\text { produce la institución } \\
\text { educativa y la emplea como } \\
\text { insumo en la toma de } \\
\text { decisiones institucionales en } \\
\text { favor de la mejora de los } \\
\text { aprendizajes }\end{array}$ & $\begin{array}{l}\text { - Desarrolla mecanismos que facilitan la generación y el acceso del } \\
\text { conocimiento por parte de los actores educativos y que permiten } \\
\text { compartir la información de la vida de la escuela generada a través } \\
\text { de diversas fuentes. } \\
\text { - Impulsa una cultura organizativa orientada a compartir el } \\
\text { conocimiento y el trabajo cooperativo en favor de la mejora } \\
\text { continua. } \\
\text { - Utiliza herramientas pertinentes y oportunas de procesamiento y } \\
\text { organización de la información que contribuyen con la toma de } \\
\text { decisiones institucionales en favor de la mejora de los aprendizajes. } \\
\text { Sistematiza la información presentada por los diversos equipos de } \\
\text { trabajo institucionales acerca de la marcha de la gestión escolar. }\end{array}$ \\
\hline & $\begin{array}{l}\text { 13. Implementa estrategias y } \\
\text { mecanismos de transparencia y } \\
\text { rendición de cuentas de la } \\
\text { gestión escolar ante la } \\
\text { comunidad educativa. }\end{array}$ & $\begin{array}{l}\text { - } \begin{array}{l}\text { Asume la importancia de hacer públicos los logros de la institución } \\
\text { - }\end{array} \text { Diseña, organiza e implementa diversos mecanismos de } \\
\text { transparencia y rendición de cuentas como exposiciones, } \\
\text { reuniones, paneles, etc. -que involucran a los padres de familia- } \\
\text { para hacer públicos los resultados de la escuela y los aprendizajes } \\
\text { de los estudiantes. } \\
\text { - Implementa algunos mecanismos para obtener los puntos de vista } \\
\text { de la comunidad, utilizando la información recogida para mejorar } \\
\text { la gestión de la escuela } \\
\text { Desarrolla acciones destinadas a evitar situaciones que propicienla } \\
\text { corrupción (uso indebido de cargos, venta de notas, nepotismo, } \\
\text { impunidad, entre otras). } \\
\text { Promueve procesos de reflexión conjunta en la comunidad } \\
\text { educativa sobre la corrupción y sus consecuencias a nivel de } \\
\text { escuela, localidad y país, promoviendo una cultura de } \\
\text { transparencia. }\end{array}$ \\
\hline
\end{tabular}




\section{Resultados}

Como el objetivo propuesto fue determinar las características del liderazgo pedagógico de los directivos a partir de una escala de autovaloración de sus competencias, se establecieron cuatro niveles en función de dos competencias (mencionadas en la Tabla 1) establecidas en el Marco de Buen Desempeño Directivo.

La primera competencia (Tabla 2), está referida a cómo conduce la planificación institucional a partir del conocimiento de los procesos pedagógicos, el clima escolar, las características de los estudiantes y su entorno, orientándola hacia el logro de metas de aprendizajes; y, la segunda, apunta a si lidera procesos de evaluación de la gestión de la institución educativa y de rendición de cuentas, en el marco de la mejora continua y el logro de aprendizajes (Tabla 3).

Los niveles que se hallaron fueron cuatro:

- Nivel Inicio: comprende a directivos que no están asumiendo su liderazgo pedagógico en la planificación democrática ni en la ejecución de los instrumentos de gestión. Está compuesto por 2 directivos que hacen el $2.92 \%$ de los investigados.

- Nivel Proceso: comprende a directivos que están asumiendo su liderazgo pedagógico, pero con deficiencias. Está compuesto por 12 directivos que representan el 20.8\% del total estudiado.

- Nivel Logrado: comprende a directivos que están asumiendo su liderazgo pedagógico de manera adecuada, compuesta por 28 directivos que representan el $46.5 \%$ de los investigados.

- Nivel Destacado: comprende a directivos que están asumiendo plenamente su liderazgo pedagógico, formado por 18 directivos que representa el $29.8 \%$ de los directivos investigados.

Luego, se determinó de manera más detallada cada uno de los desempeños valorados según la competencia respectiva. De esta manera, en cuanto a la competencia "conduce la planificación institucional", se ponderaron dos desempeños. Se halló que los resultados son homogéneos en el desempeño "Diagnostica las características del entorno institucional, familiar y social que influyen en el logro de las metas de aprendizaje", ubicándose en el nivel logrado con los siguientes detalles en los sub desempeños:

- $\quad 61.67 \%$ de directivos cumplen el sub desempeño "Caracterizo las condiciones de mi institución educativa para el diseño de los objetivos institucionales".

- $\quad 50.00 \%$ de directivos cumplen el sub desempeño "Utilizo la información del diagnóstico como insumo en la planificación escolar".

Sobre la ponderación al desempeño "Diseña de manera participativa los instrumentos de gestión escolar teniendo en cuenta las características del entorno institucional, familiar y social, estableciendo metas de aprendizaje", también se ubica en el nivel logrado, aunque en una dimensión se encuentra en proceso, tal como se aprecia:

- $\quad$ 58.33\% de directivos cumplen el sub desempeño "Establezco la gestión en función del logro de aprendizaje de los estudiantes de mi institución educativa”. 
- $\quad 53.33 \%$ de directivos se encuentran en proceso de lograr el sub desempeño "Convoco a los padres de familia o estudiantes para ajustar los instrumentos de gestión escolar", la dimensión con mayor deficiencia.

- $\quad 53.33 \%$ de directivos cumplen el sub desempeño "Desarrollo mecanismos de acceso a la información de mi IE de los actores educativos".

- $\quad 41.67 \%$ de directivos se ubican en el nivel destacado en el sub desempeño "Convoco a los docentes de mi IE para ajustar los instrumentos de gestión escolar".

Tabla 2

Resultados del liderazgo pedagógico asociados a la conducción de la planificación institucional

\begin{tabular}{|c|c|c|c|c|c|c|c|c|c|c|c|}
\hline \multirow{2}{*}{$\mathbf{N}^{\circ}$} & \multirow{2}{*}{ Considero que como directivo } & \multicolumn{2}{|c|}{ Nunca } & \multicolumn{2}{|c|}{ A veces } & \multicolumn{2}{|c|}{$\begin{array}{l}\text { Casi } \\
\text { siempre }\end{array}$} & \multicolumn{2}{|c|}{ Siempre } & \multicolumn{2}{|c|}{ Total } \\
\hline & & $\mathbf{f}$ & $\%$ & $f$ & $\%$ & $\mathbf{f}$ & $\%$ & $f$ & $\%$ & $f$ & $\%$ \\
\hline 1 & $\begin{array}{l}\text { Identifico las características más } \\
\text { importante de mi IE }\end{array}$ & 0 & 0.00 & 6 & 10.00 & 28 & 46.67 & 26 & 43.33 & 60 & 100.00 \\
\hline 2 & $\begin{array}{l}\text { Utilizo la información del diagnóstico } \\
\text { como insumo en la planificación escolar }\end{array}$ & 1 & 1.67 & 6 & 10.00 & 30 & 50.00 & 23 & 38.33 & 60 & 100.00 \\
\hline 3 & $\begin{array}{l}\text { Realizo el análisis FODA de mi IE para la } \\
\text { mejora de los procesos pedagógicos }\end{array}$ & 1 & 1.67 & 10 & 16.67 & 29 & 48.33 & 20 & 33.33 & 60 & 100.00 \\
\hline 4 & $\begin{array}{l}\text { Caracterizo las condiciones de mi IE } \\
\text { para el diseño de los objetivos } \\
\text { institucionales }\end{array}$ & 2 & 3.33 & 6 & 10.00 & 37 & 61.67 & 15 & 25.00 & 60 & 100.00 \\
\hline 5 & $\begin{array}{l}\text { Convoco a los docentes de mi IE para } \\
\text { ajustar los instrumentos de gestión } \\
\text { escolar }\end{array}$ & 2 & 3.33 & 11 & 18.33 & 22 & 36.67 & 25 & 41.67 & 60 & 100.00 \\
\hline 6 & $\begin{array}{l}\text { Convoco a los padres de familia o } \\
\text { estudiantes para ajustar los } \\
\text { instrumentos de gestión escolar }\end{array}$ & 3 & 5.00 & 32 & 53.33 & 17 & 28.33 & 8 & 13.33 & 60 & 100.00 \\
\hline 7 & $\begin{array}{l}\text { Formulo -en consenso con los actores- } \\
\text { la visión, misión y metas institucionales } \\
\text { y de aprendizaje }\end{array}$ & 2 & 3.33 & 12 & 20.00 & 26 & 43.33 & 20 & 33.33 & 60 & 100.00 \\
\hline 8 & $\begin{array}{l}\text { Establezco la gestión en función del } \\
\text { logro de aprendizaje de los estudiantes } \\
\text { de mi IE }\end{array}$ & 0 & 0.00 & 5 & 8.33 & 35 & 58.33 & 20 & 33.33 & 60 & 100.00 \\
\hline 9 & $\begin{array}{l}\text { Comunico o difundo el contenido de los } \\
\text { instrumentos de gestión escolar }\end{array}$ & 3 & 5.00 & 13 & 21.67 & 30 & 50.00 & 14 & 23.33 & 60 & 100.00 \\
\hline 10 & $\begin{array}{l}\text { Desarrollo mecanismos de acceso a la } \\
\text { información de mi IE de los actores } \\
\text { educativos }\end{array}$ & 2 & 3.33 & 17 & 28.33 & 32 & 53.33 & 9 & 15.00 & 60 & 100.00 \\
\hline
\end{tabular}

En cuanto a la competencia "Lidera procesos de evaluación de la gestión de la institución educativa", se halló que los resultados son también homogéneos en dos desempeños en el nivel logrado, pero seguido de en proceso. El desempeño gestiona la información que produce la institución educativa y la emplea; comprende cinco en el sub desempeño ubicado en logrado, pero seguido de en proceso:

- $\quad 53.33 \%$ de directivos se ubican en el nivel logrado en el sub desempeño "utilizo herramientas de procesamiento y organización de la información que sirvan a la toma de decisiones institucionales". 
- $\quad 50.00 \%$ de directivos se ubican en el nivel logrado en el sub desempeño "asumo la importancia de hacer públicos los logros de la IE".

- $\quad 38.33 \%$ de directivos se ubican en el nivel de proceso en el sub desempeño "comparto información de la vida de mi institución a través de diversas fuentes".

En cuanto al desempeño "Implementa estrategias y mecanismos de transparencia y rendición de cuentas de la gestión escolar ante la comunidad educativa” se halló:

- $\quad 53.33 \%$ de directivos se ubican en el nivel destacado en el sub desempeño "promuevo una cultura de transparencia en mi institución".

- $\quad 51.67 \%$ de directivos en el nivel logrado en el sub desempeño "lidero mecanismos de transparencia y rendición de cuentas sobre los aprendizajes de los estudiantes de mi institución educativa".

- $\quad$ 43.33\% de directivos en el nivel en proceso en el sub desempeño "implemento mecanismos para recoger opinión de la comunidad en la gestión de mi escuela".

Tabla 3

Resultados del liderazgo pedagógico en la evaluación de la gestión

\begin{tabular}{|c|c|c|c|c|c|c|c|c|c|c|c|}
\hline \multirow{2}{*}{$\mathbf{N}^{\circ}$} & \multirow{2}{*}{ Considero que como directivo } & \multicolumn{2}{|c|}{ Nunca } & \multicolumn{2}{|c|}{ A veces } & \multicolumn{2}{|c|}{ Casi siempre } & \multicolumn{2}{|c|}{ Siempre } & \multicolumn{2}{|c|}{ Total } \\
\hline & & f & $\%$ & $f$ & $\%$ & $f$ & $\%$ & f & $\%$ & f & $\%$ \\
\hline 1 & $\begin{array}{l}\text { Comparto información de la vida } \\
\text { de mi IE a través de diversas } \\
\text { fuentes }\end{array}$ & 6 & 10.00 & 23 & 38.33 & 24 & 40.00 & 7 & 11.67 & 60 & 100.00 \\
\hline 2 & $\begin{array}{l}\text { Impulso una cultura organizativa } \\
\text { para compartir conocimiento de la } \\
\text { IE }\end{array}$ & 2 & 3.33 & 18 & 30.00 & 31 & 51.67 & 9 & 15.00 & 60 & 100.00 \\
\hline 3 & $\begin{array}{l}\text { Utilizo herramientas de } \\
\text { procesamiento y organización de } \\
\text { la información que sirvan a la toma } \\
\text { de decisiones institucionales. }\end{array}$ & 2 & 3.33 & 15 & 25.00 & 32 & 53.33 & 11 & 18.33 & 60 & 100.00 \\
\hline 4 & $\begin{array}{l}\text { Sistematizo la información de los } \\
\text { equipos de mi IE sobre la gestión } \\
\text { escolar }\end{array}$ & 2 & 3.33 & 11 & 18.33 & 29 & 48.33 & 18 & 30.00 & 60 & 100.00 \\
\hline 5 & $\begin{array}{l}\text { Asumo la importancia de hacer } \\
\text { públicos los logros de la IE. }\end{array}$ & 2 & 3.33 & 10 & 16.67 & 30 & 50.00 & 18 & 30.00 & 60 & 100.00 \\
\hline 6 & $\begin{array}{l}\text { Lidero mecanismos de } \\
\text { transparencia y rendición de } \\
\text { cuentas sobre los aprendizajes de } \\
\text { los estudiantes de mi IE }\end{array}$ & 1 & 1.67 & 5 & 8.33 & 31 & 51.67 & 23 & 38.33 & 60 & 100.00 \\
\hline 7 & $\begin{array}{l}\text { Implemento mecanismos para } \\
\text { recoger opinión de la comunidad } \\
\text { en la gestión de mi IE }\end{array}$ & 1 & 1.67 & 26 & 43.33 & 26 & 43.33 & 7 & 11.67 & 60 & 100.00 \\
\hline 8 & $\begin{array}{l}\text { Desarrollo acciones para evitar } \\
\text { situaciones de corrupción en la IE }\end{array}$ & 1 & 1.67 & 14 & 23.33 & 20 & 33.33 & 25 & 41.67 & 60 & 100.00 \\
\hline 9 & $\begin{array}{l}\text { Promuevo la reflexión de la } \\
\text { comunidad educativa sobre los } \\
\text { efectos de la corrupción. }\end{array}$ & 2 & 3.33 & 17 & 28.33 & 21 & 35.00 & 20 & 33.33 & 60 & 100.00 \\
\hline
\end{tabular}




$\begin{array}{lllllllllllll}10 & \begin{array}{l}\text { Promuevo una cultura de } \\
\text { transparencia en mi IE }\end{array}\end{array} \quad$\begin{tabular}{llllllllll} 
& 0.00 & 5 & 8.33 & 23 & 38.33 & 32 & 53.33 & 60 & 100.00 \\
\hline
\end{tabular}

\section{Discusión}

La investigación ratifica las características ideales del liderazgo distribuido y transformacional en directivos como factor clave para las mejoras esperadas de las instituciones educativa (Lorenzo, 2005). No es nuevo reconocer la importancia del directivo, pero sí es importante una valoración a partir de los propios directores y subdirectores de escuelas estatales.

La investigación se enmarca en los estudios empíricos para profundizar en el mayor conocimiento de las características que poseen los gestores de las escuelas, en cuanto a sus competencias y desempeños del ejercicio de su liderazgo pedagógico. Siguiendo el objetivo de la investigación, se ha tomado como referente el primer dominio del Marco de Buen Desempeño Directivo de Perú: "Gestión de las condiciones para la mejora de los aprendizajes". Abarca las competencias que lleva a cabo el directivo para construir e implementar la reforma de la escuela, gestionando las condiciones para la mejora de aprendizajes a través de la planificación, la promoción de la convivencia democrática e intercultural y la participación de las familias y comunidad (competencia 1) y, evaluando sistemáticamente la gestión de la institución educativa en dos competencias (competencia 4). Asimismo, considera cuatro desempeños y veinte sub desempeños materializados en cada uno de los ítems.

Se ha destacado que un gestor escolar con liderazgo pedagógico debe poseer varias competencias con énfasis en aspectos de participación de la comunidad escolar, como capacidades humanas; ello fortalece el trabajo colegiado de los docentes en aspectos tales como la confianza, el ánimo y la toma de decisiones (Medina, 2013).

Los resultados muestran que el liderazgo pedagógico directivo, en cuanto a la participación para el diseño y la implementación de los instrumentos de gestión se ubican en el nivel "logrado" en 28 directivos que representan el $46.5 \%$ de los sujetos; seguidos del nivel destacado con 18 directivos que representa el $29.8 \%$ de los directivos estudiados. Se evidenció, además, deficiencias en el desempeño relacionado con diseñar de manera participativa los instrumentos de gestión escolar con el aporte y perspectiva de los padres de familia como de los estudiantes. También, se halló limitaciones en el desempeño referido a "gestiona la información que produce la institución educativa y la emplea como insumo en la toma de decisiones institucionales en favor de la mejora de los aprendizajes".

Lo encontrado denotaría que hay deficiencias en el liderazgo pedagógico declarado por los propios directivos. Esto, coincide con el trabajo cualitativo desarrollado por Vela-Quico y Villalba (2019) donde se investigó - en directivos- las teorías implícitas para el ejercicio del liderazgo pedagógico y en donde los sujetos expresaron el estar más concentrados en lo administrativo y en descuido de los aspectos pedagógicos.

Del mismo modo, este esfuerzo investigativo halló que, en la autovaloración de los directivos, reconocen deficiencias importantes en los sub desempeños relacionados con el uso de la información y en la toma de decisiones de la gestión escolar. Los directivos tienen niveles inferiores en compartir información de la vida escolar a través de diversas fuentes (38.33\%), impulsar una cultura organizativa para compartir conocimiento de la escuela (30.00\%) o utilizar herramientas de procesamiento y organización de la información que sirvan a la toma de decisiones institucionales (25.00\%). 
Datos análogos son hallados por Estefanía (2009) en los directivos de escuelas en el uso de los resultados de la Evaluación Censal de Estudiantes; una prueba nacional para valorar aprendizajes en comunicación y matemática de los estudiantes de sus escuelas que debería repercutir tanto en el ámbito pedagógico como la gestión institucional. A mismo tiempo, este estudio confirma los estudios de Bolívar, Caballero y García-Garnica (2017) que nos habla de que el no procesamiento adecuado de los resultados de su propia escuela constituye un impedimento para liderar una comunidad de aprendizaje centrada en los aprendizajes.

Por último, cabe indicar, que este estudio no supone un menosprecio a lo administrativo, sino más bien, su ubicación en el sitio que corresponde. Al mismo tiempo, confirma el trabajo la necesidad de consolidar en los directivos el liderazgo transformacional y facilitar la alofilia (sentimientos positivos hacia el grupo) que se traduce en competencias humanas como la empatía, el trabajo en equipo, la delegación de funciones, la escucha activa y la implicación de la comunidad, entre otros (Gómez y Medina, 2014). Pero, sobre todo, consideramos la necesidad de profundizar en este objeto de estudio con aproximaciones que trasciendan (o complementen) la percepción de los propios directivos como en nuestro caso. Para esta empresa, es deseable el incluir a los profesores, padres de familia y estudiantes bajo diseños mixtos de investigación capaces de atender a aproximaciones cuantitativas como cualitativas.

\section{Referencias}

Barber, M., y Mourshed, M. (2007). How the world's best-performing schools systems come out on top. McKinsey y Company.

Bolívar, A. (2010). Liderazgo para el aprendizaje. OGE, (1), 15-20. Recuperado de https://educrea.cl/wpcontent/uploads/2019/03/DOC1-Lid-Aprendizaje.pdf

Bolívar, A., Caballero, K., y García-Garnica, M. (2017). Evaluación multidimensional del liderazgo pedagógico: claves para la mejora escolar. Ensaio: Avaliação e Políticas Públicas em Educação, 25(95), 483-506. doi: 10.1590/S0104-40362017002500780

Bolívar, A., López, J., y Murillo, F. J. (2013). Liderazgo en las instituciones educativas: Una revisión de líneas de investigación. Revista fuentes, 14, 15-60. Recuperado de http://institucional.us.es/revistas/fuente/14/Firma\%2oinvitada.pdf

Estefanía, M. T. (2009). Informe final. Uso de informes ECE 2009-2010. MINEDU.

Ganga-Contreras, F., Villegas-Villegas, F., y Pedraja-Rejas, L. (2016). Transformational leadership and its effect on teaching management: The case of aschool in northern Chile. Interciencia 41 (9), 596604. Recuperado de: https://www.interciencia.net/volumen-41/numero-09-5/

García Garduño, J. M., Slater, C. L., y López Gorosave, G. (2010). El director escolar novel de primaria: problemas y retos que enfrenta en su primer año. Revista mexicana de investigación educativa, 15(47), 1051-1073. Recuperado de: https://www.comie.org.mx/revista/v2018/rmie/index.php/nrmie/article/view/469

Gómez, R. M., y Medina, A. (2014). El liderazgo pedagógico: competencias necesarias para desarrollar un programa de mejora en un centro de educación secundaria. Perspectiva Educacional, 53(1), 91-113. Recuperado de http://www.perspectivaeducacional.cl/index.php/peducacional/article/view/127

Hernández, R. Fernández, C. y Baptista, P. (2003). Metodología de la investigación. México: McGraw-Hill.

Leithwood, K. A., Begley, P. T., y Cousins, J. B. (1990). The Nature, Causes and Consequences of Principals' Practices: An Agenda for Future Research. Journal of educational administration, 28(4). doi: 10.1108/09578239010001014

Lorenzo, M. (2005). El liderazgo en las organizaciones educativas: revisión y perspectivas. Revista Española de Pedagogía, LXIII(232), 267-388. Recuperado de: https://revistadepedagogia.org//xiii/no-232/el-liderazgo-en-las-organizaciones-educativasrevision-y-perspectivas-actuales/101400010268/

Medina, C. (2013). Formación de líderes, inteligencia emocional y formación del talento. Madrid: Universitas. 
MINEDU (2014). Marco de Buen Desempeño Directivo. Lima: MINEDU.

MINEDU (2019). R. V. M. N 011-2019-MINEDU Instrumentos de gestión escolar. Lima: MINEDU.

Soto, A. C., Torres, J. M. T., y Barboza, E. C. (2019). Liderazgo distribuido en los institutos de educación secundaria de Melilla, España. Revista de ciencias sociales, 25(3), 42-58.

Vela-Quico, Gabriel y K. Villalba. (2019). Implicit Theories of Directors in the Exercise of Pedagogical Leadership. International Journal of Recent Technology and Engineering (IJRTE), 522-528. 